\title{
Posterior Knee Arthrolysis: About 08 Cases
}

Bensassi Achraf*, El Ghadraoui Redouane, Zahraoui Anass, Abid Hatim, Mohammed El Idrissi, Abdelhalim Elibrahimi, Abdelmajid Elmrini

Department of Osteo-Articular Surgery B4, Hassan II CHU, University Sidi Mohammed Ben Abdellah of Fez, Morocco

DOI: $\underline{10.36347 / \text { sasjs.2020.v06i04.010 }}$

| Received: 14.04.2020 | Accepted: 22.04.2020 | Published: 24.04.2020

*Corresponding author: Bensassi Achraf

Abstract

Original Research Article

Post-traumatic stiffness of the knee is a common complication. It can be in flexion, extension, or mixed. It can be of intra-articular and / or extra-articular origin, and the surgical treatments available are numerous. These can be performed in the open or under arthroscopy. Understanding and analyzing the causes of this stiffness is essential. In general, flexion deficits are due to a retraction of the anterior structures and / or a posterior stopper. Deficits of extension rather by a posterior retraction and / or an anterior stop. We report a series of 8 cases of knee stiffness operated in the osteoarticular surgery department 2 of CHU HASSAN II of FES between January 2009 and December 2016. The objective of our study is to determine the epidemiological profile of these stiffnesses, the causes of these stiffness, the effectiveness of the surgical treatment evaluated by the IKS score, the gain in mobility and the causes of failure.

Keywords: Knee, arthrolysis, stiffness.

Copyright @ 2020: This is an open-access article distributed under the terms of the Creative Commons Attribution license which permits unrestricted use, distribution, and reproduction in any medium for non-commercial use (NonCommercial, or CC-BY-NC) provided the original author and source are credited.

\section{INTRODUCTION}

Knee stiffness is a possible complication of any joint and / or extra-articular trauma. It can be divided into three entities: limitation of flexion, extension, mixed limi-tation (interesting flexion and extension) compared to the healthy contralateral side if it is. This stiffness has two components: articular: due to tissue changes; and extra-articular: due to the adhesion of the quadriceps to a possible femoral callus, to the aponeurosis of the femur and to the intermuscular partitions ...

Many surgical techniques have been proposed for more than 30 years, in the open and then under arthroscopy, in order to best treat all the causes of stiffness while reducing morbidity $[1,2]$.

\section{MATERIAL AND METHODS}

Between January 2009 and December 2016, a retrospective study was carried out, concerning 08 cases of post traumatic and non-traumatic knee arthrolysis, collected in the osteo-articular surgery department B4 of FEZ -MOROCCO. An operating sheet was established for each patient to facilitate the collection and analysis of the different epidemiological, clinical, radiological, therapeutic and progressive parameters.

\section{RESULTS}

At the end of the results we found that the age of our patients varies between 18 to 43 years, with an average age of 31 years.In our series, we note a female predominance, it is 3 men for 5 women. The right side was the most operated with 5 cases against 3 cases for the left side. The traumatic context represents half of the etiologies of flexion stiffness. The duration of progression to stiffness varies between 6 months and 14 years with an average of 60 months. The treatment for stiffness in flexion of the knee consisted of a posterior open arthrolysis.

We evaluated the functional results of the operated knees, based on 4 parameters: pain, mobility, stability and walking distance.All patients benefited from a pre- and post-operative assessment using the IKS score.The post-operative results were generally satisfactory with an average final gain of $42.5^{\circ}$.

\section{DISCUSSION}

Knee stiffness is defined as a passive limitation of the range of motion in the joint [4-6]. It is the most frequent complication of knee trauma [7-9] given the negligence of some of our patients after the treatment of the initial lesions and the problem of access to rehabilitation in our context [10, 14]. Age, gender, dominance and seniority did not influence the severity of the stiffness. 
In the literature it is partially young and active subjects with an average age which varies according to the series between 37 [13] and 44 years [15], this because young subjects are more exposed to trauma, to work accidents, or sports accidents which are the primary cause of stiffness. The various series in the literature show that there is a predominance in male patients, unlike ours where the male / female ratio is 0.6 .

The duration of stiffness evolution is closely linked to the gain obtained during initial rehabilitation and depends on the socio-economic context of the patients.

Regarding the etiologies of stiffness, there are predisposing factors which have been reported in the literature [13]: joint infection; prolonged postoperative immobilization and ill-adapted rehabilitation; the most common knee fractures causing stiff knees are fractures of the lower end of the femur; knee surgery: more recently, post-operative monitoring of operated knees has made it possible to estimate $11 \%$ of patients at risk of stiffness (existence of scar problem, superficial or deep sepsis, algodystrophy, non-specific inflammatory reaction. ..) and other etiologies: Rehabilitation error, defensive muscular contracture, non-traumatic causes (gonarthrosis...).

The surgical treatments available are numerous. These can be performed in the open or under arthroscopy.

Arthroscopic arthrolysis is conventionally reserved for limited stiffness, of intra-atrial origin, with a joint chamber sufficient to return safely to the knee joint because of the risks of vasculo-nerve damage existing with this technique. The decrease in intra articular capacity, the thickened capsule, adhesions, modification of the anatomical vasculo-nervous relationships make this intervention particularly delicate. It is more difficult technically, and requires sufficient control of the posterior arthroscopic pathways $[11,12]$.

The standard anterior arthroscopic pathways are performed, and the anterior release is performed systé $\neg$ matically in the first place. It must be checked that there is no extension of conflict in the notch. The scope is then introduced by the anterolateral route under the posterior cruciate ligament (LCP) to the posteromedial space. A post-medial approach is carried out by trans-illumination with the needle. The optics are then directed upwards. The posterior capsule and the adhesions are sectioned flush with the femur using a motorized resector or by an electrocoagulation probe, until individualizing the muscle fibers of the medial gastrocne $\neg$ median muscle.

As with the open technique, the knee is placed in extension to assess the effectiveness of this release. If it is not enough, a posterolateral approach is performed back and forth from the posteromedial approach and through the intercondylar septum above the LCP, at the level of the condyles, knee bent at $90^{\circ}$. This technique was described by $\mathrm{Ph}$. Beaufils in 2003 [11] and it is harmless if it is properly mastered. The septum is excised, making access to the posterolateral compartment easier. The capsule and the lateral condylar shell are sectioned flush with the femur according to the same methods as previously (Figure-1).

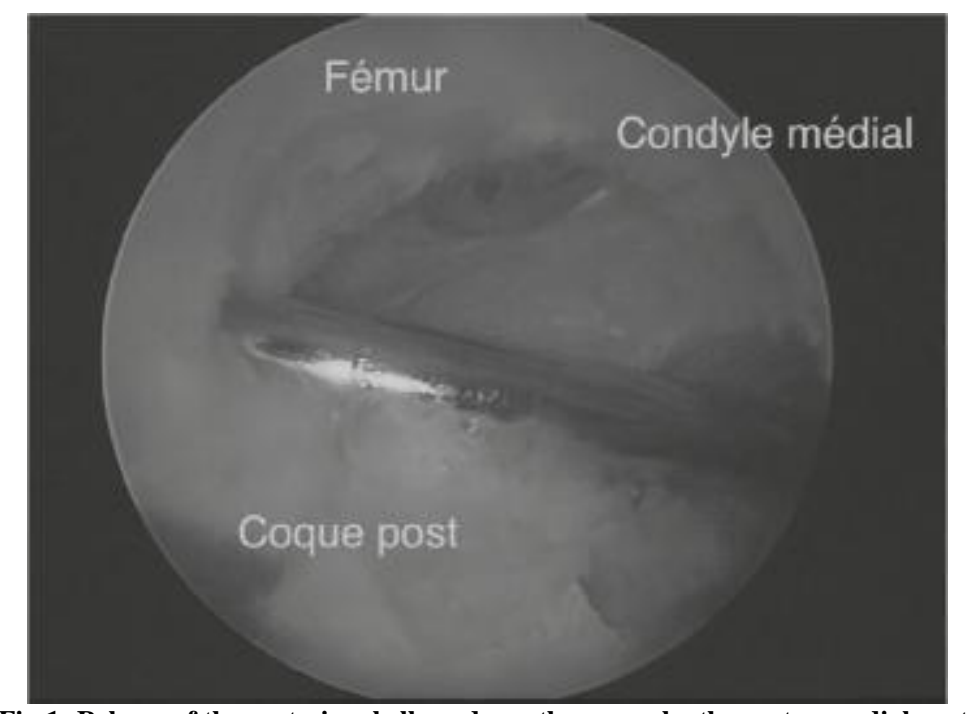

Fig-1: Release of the posterior shells under arthroscopy by the posteromedial route

The gastrocnemius muscles can also be released at this stage under arthroscopy. Rather, it is an opening of the deep side of their fascia. A gentle mobilization ends the intervention. The incisions are closed with intra-articular drainage. 
Arthroscopic arthrolysis, despite its invasive nature, has proved less traumatic and constitutes an alternative, on the one hand to mobilization under general anesthesia in recent stiffness, on the other hand to open arthrolysis in stiffness older. All studies agree on the superiority of percutaneous arthrolysis under arthroscopic control over other techniques, with the advantages that we usually recognize in arthroscopic techniques : reduction in morbidity and in particular the risk of infection, rapidity of follow-up operating, functional results are more or less equivalent or better in comparison with the other mobilizing techniques in knee stiffness (mobilization under general anesthesia and surgical arthrolysis.

The essential principles in post-operatoi are: to limit the risk of postoperative hematoma (by keeping the Redon drain long enough (at least 2 days), with a slightly compressive bandage at the start, of cryotherapy every day...), because the risk of bleeding is high and the cicatricial fibrosis induced by the resorption of a hematoma makes run a risk of recurrence of the stiffness to start the rehabilitation by intermittent mobilization (as of the arrival of the patient in the recovery room), and to transfer the patient in a rehabilitation center on the 3rd postoperative day. To fight pain effectively. They all aim to oppose the risk of a reduction in the amplitude gain obtained during the mobilizing intervention ; their importance is equivalent to that of the surgical technique.

As for the open posterior arthrolysis which is a very elective way for the repair of the posterior cruciate ligament not risking damaging the vascularization of the latter, is based on a postero-medial retro ligament vertical arthrotomy (Figure-2).

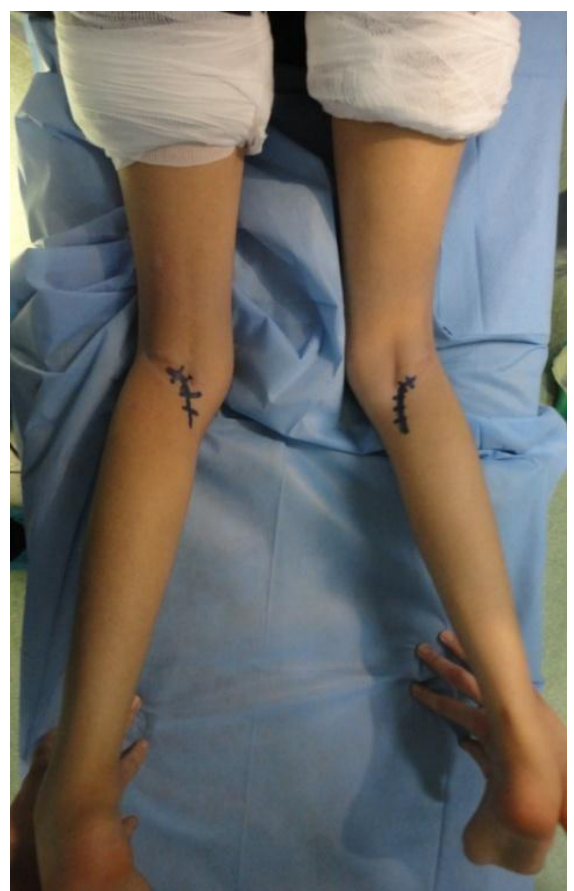

Fig-2: Posterior approach to the knee
The installation assumes that the anterior arthrolysis has been carried out beforehand, if it was necessary to obtain sufficient flexion and posterior space and to remove the vascular-nervous structures. The medial shell is individualized from the femur, and sectioned flush with it. The posterior vascular-nervous elements are at a respectable distance (more than $2 \mathrm{~cm}$ ) when the knee is bent at $90^{\circ}$ and that one remains flush with the posterior surface of the femur. The knee is then placed in extension to determine the effectiveness of this release. If it is insufficient, a $4 \mathrm{~cm}$ retro-ligament lateral approach, above the head of the fibula and in front of the biceps femoris tendon is performed. The capsule is incised close to the femur, after a retrocondylar arthrotomy. The lateral shell is then completely removed from the posterior surface of the femoral metaphysis. At this stage, a progressive mobilization can be carried out to complete the gain in mobility. The fascia of the gastrocnemius muscles, or even the tendon insertions on the femur can be cut at this stage if necessary (Figure-3).
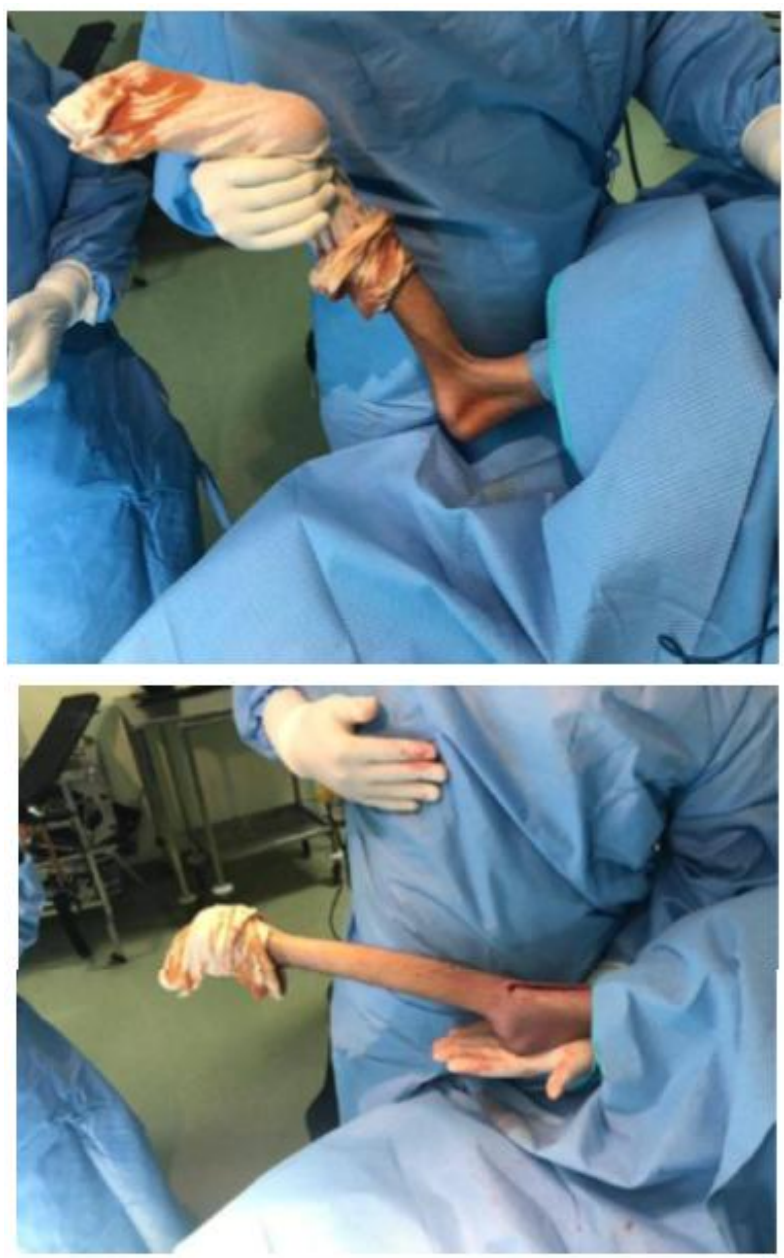

Fig-3: Shows intraoperative gain for posterior knee release in a patient with stiff knee flexion

The incisions are closed, with an intra-articular drain kept for at least 2 days (depending on the bleeding) and rehabilitation is undertaken immediately. This technique has few drawbacks apart from the 
section of the internal twin, but if it is carefully repaired, this seems to be without drawback. You can also simply roam its upper insertion. The quality of postoperative management of knee arthrolysis is essential to obtaining a satisfactory functional result.

Rehabilitation takes a prominent place and Merle d'Aubigné and Benassy point out that "if we can assess the usefulness of the surgical procedure at $50 \%$, the importance of rehabilitation is at least - equal". The purpose here is not to detail a specific reeducation protocol, but to express its main principles by emphasizing a few fundamental points.

The rehabilitation of arthrolyses must therefore be immediate, right out of the block for Judet, thus avoiding fibrosis and the vicious circle of stiffness. This early management is all the more necessary as there are cartilage lesions. It includes alternating knee postures in flexion and extension, associated with manual and / or mechanical mobilization (arthromotor).

\section{CONCLUSION}

We can conclude the importance of preventing knee stiffness since it affects the patient's functional prognosis and has an impact on his socio-professional life.The diagnosis of stiffness is based on the clinic and must be supplemented by radiological examinations. The treatment has several components. In our study, open posterior arthrolysis and arthroscopic arthrolysis are the most used treatments. Arthroscopic and open techniques can be combined in a thoughtful surgical strategy to treat the different components of stiffness in stages, in one step.

\section{REFERENCES}

1. Trickey EL. Rupture of the posterior cruciate ligament of the knee. The Journal of bone and joint surgery. British volume. 1968 May;50(2):334-41.

2. Garotta L, Dejour D, Sadile F. Les "clunk" syndromes 8 emes journées lyonnaises de chirurgie du genou 6-7-8, 1995 April.

3. Kerboul M, Deburge A. Etiologie traumatique des raideurs du genou ; raideurs et arthrolyses post traumatique du genou Rapport annuel XLVème congrès SOFCOT.

4. Allieu Y. Raideur et arthrolyse du genou. Rev Chir Orthop, 1989;1:157-166.

5. Herweig S, De Smet L. Operative treatment of knee stiffness: evaluation and outcome. Acta Orthop Belg, 2003;69:18-22.

6. Gill GS, Joshi AB. Long-term results of cemented, posterior cruciate ligament-retaining total knee arthroplasty in osteoarthritis. The American journal of knee surgery. 2001;14(4):209-14.

7. Garcia RM, Hardy BT, Kraay MJ, Goldberg VM. Revision total knee arthroplasty for aseptic and septic causes in patients with rheumatoid arthritis. Clinical Orthopaedics and Related Research®. 2010 Jan 1;468(1):82-89.

8. Gallet. Anesthésie pour prothèse totale du genou: PEC médicale en période périopératoire. 10, 2007/2-287-30285-9-24.

9. Insall JN. Surgery of the knee New York: Churchill Livingstone, 1993.

10. Badet R. Les fractures de l'extrémité inférieure du fémur; mécanismes et classification DIU de pathologie du genou (Bercovi M, Goutallier D, Moyen B, Neyret P, Saragaglia D), 2000 May.

11. Heuleu JN, Et Neyret P, Montero C, Petit H, Peyre M, Middleton JH, Jaeger G, LE BLAY, Th. JUDET, G. DESCHAMPS, H. JUDET, Breton G. Conduite à tenir devant les raideurs postopératoires du genou. Table ronde SO.F.C.O.T. SOFMER, novembre 2001

12. Brunet JC. Traitement des raideurs du genou par libération de l'appareil extenseur. Ann Med Phys, 1978;21:45-57.

13. Hulet C, Djian P. Stiffness of the knee joint--SFA 2002. Revue de chirurgie orthopedique et reparatrice de l'appareil moteur. 2007 Dec;93(8 Suppl):5S14-5.

14. Venet G, Gouin F, Legeay O, Huguet D, Passuti N. Percutaneous arthrolysis of the knee ; technique, indications and results Clinique Chirurgicale Orthopédique, Pôle ostéoarticulaire.

15. Rim El Fagour. Raideur du genou; CHU IBN SINA RABAT thèse $\mathrm{N}^{\circ}$ 203/1. 